\title{
A Note on Finite Difference Methods for Solving the Eigenvalue Problems of Second-Order Differential Equations
}

\author{
By M. R. Osborne
}

1. Introduction. In many cases of importance a finite difference approximation to the eigenvalue problem of a second-order differential equation reduces the problem to that of solving the eigenvalue problem of a tridiagonal matrix having the Sturm property. In this paper we illustrate the use of the Rayleigh quotient for obtaining a quadratically convergent iteration to the eigenvalues of such a matrix.

If the finite difference approximation is to give a tridiagonal matrix having the Sturm property then there must be restrictions on the coefficients in the differential equation and on the form of the boundary conditions. The type of problem we consider is defined in Section 2.

The Rayleigh quotient iteration is derived in Section 3 where we also relate it to a class of quadratically convergent iterations. This section is included at the suggestion of a referee to whom I am indebted for several other constructive suggestions and, in particular, for bringing to my attention the paper by J. W. Cooley [1].

The Rayleigh quotient iteration is exemplified in Section 4. We show that it can be conveniently linked to a difference correction procedure for estimating the error in the solution of the finite difference approximation. The stability of the iteration is discussed in Section 5; and a routine which we have used on an electronic computer is described in Section 6 .

2. Finite Difference Approximations. In the usual notation the standard method of approximating to a second-order differential equation using finite difference formulas on a grid of equispaced points equates $h^{2} \frac{d^{2}}{d x^{2}}$ with $\delta^{2}$, and $h \frac{d}{d x}$ with $\mu \delta$ where $h$ is the grid spacing. For example, the equation

$$
\frac{d^{2} y}{d x^{2}}+p(x) \frac{d y}{d x}+(\lambda q(x)+r(x)) y=0
$$

becomes

$$
\left(\delta^{2}+h p(x) \mu \delta+h^{2}(\lambda q(x)+r(x)) y=0,\right.
$$

and when written out at the $j$ th grid point this gives

$$
\left(1-\frac{h}{2} p_{j}\right) y_{j-1}-\left(2-h^{2}\left(\lambda q_{j}+r_{j}\right)\right) y_{j}+\left(1+\frac{h}{2} p_{j}\right) y_{j+1}=0
$$

We assume boundary conditions

$$
\begin{aligned}
A y(0)+B \frac{d y(0)}{d x} & =0 \\
\text { and } y(e) & =0 .
\end{aligned}
$$

Received February 7, 1961. 
Setting $h=e / n, x_{j}=j h$ we have the finite difference approximations to (2.4) in the form

$$
\begin{aligned}
& y_{-1}=y_{1}+\alpha y_{0}, \quad \alpha=2 h \frac{A}{B}, \\
& y_{n}=0 .
\end{aligned}
$$

Combining the boundary conditions (2.5) with the set of equations (2.3) for $0 \leqq j \leqq n-1$ we obtain an algebraic eigenvalue problem which we write as

$$
\left(A+h^{2} \lambda Q\right) \mathbf{y}=0 \text {. }
$$

Here $\mathrm{y}$ is the vector with components $y_{j}, 0 \leqq j \leqq n-1, Q$ is a diagonal matrix whose $(j+1)$ st element is $q_{j}$, and $A$ is a tridiagonal matrix. We adopt the notation that the nonzero elements in the $(j+1)$ st row of $A$ are $c_{j}, a_{j}, b_{j}$.

We assume that $q(x)$ is strictly positive in $0 \leqq x \leqq e$, and that $h$ can be chosen sufficiently small so that $\left|\frac{h}{2} p_{j}\right|<1,1 \leqq j \leqq n-1$. From these assumptions it follows that the principal minors of the determinant of $A+h^{2} \lambda Q$ have the Sturm property [2, p. 181-2], so that a technique of counting signs and bisection can be used to calculate the eigenvalues of $(2.6)$.

In general the matrix $A$ is not symmetric. It can, however, be made symmetric by premultiplying it by a certain diagonal matrix $D$. If the $(j+1)$ th element of $D$ is $d_{j}$ then the elements of the $(j+1)$ th row of $D A$ are $d_{j} c_{j}, d_{j} a_{j}$, and $d_{j} b_{j}$ so that $D A$ is symmetric if $d_{j} c_{j}=d_{j-1} b_{j-1}$. This gives a recurrence for $d_{j}$ when one value which can be freely chosen is fixed. Thus we can always write equation (2.6) in the form

$$
\left(D A+h^{2} \lambda D Q\right) \mathbf{y}=0
$$

where $D A$ is symmetric, and where $D Q$ is diagonal with positive elements.

When $p(x)$ is identically zero a more accurate finite difference formula (the Numerov formula) can be used to approximate to equation (2.1). This gives the difference equations

$$
\begin{aligned}
\left(1+\frac{h^{2}}{12}\left(\lambda q_{i-1}+r_{i-1}\right)\right) y_{i-1}- & \left.\left(2-\frac{5 h^{2}}{6} \lambda q_{i}+r_{i}\right)\right) y_{i} \\
& +\left(1+\frac{h^{2}}{12}\left(\lambda q_{i+1}+r_{i+1}\right)\right) y_{i+1}=0 .
\end{aligned}
$$

In this case the appropriate boundary condition at $x=0$ is

$$
y_{-1}\left(1+\frac{h^{2}}{6}\left(\lambda q_{-1}+r_{-1}\right)\right)=y_{1}\left(1+\frac{h^{2}}{6}\left(\lambda q_{1}+r_{1}\right)\right)+\alpha y_{0}
$$

These equations combine to give the eigenvalue problem

$$
\left(M+h^{2} \lambda N\right) \text { y }=0
$$

where both $M$ and $N$ are tridiagonal matrices. It is not possible in general to make both $M$ and $N$ symmetric by premultiplying by a diagonal matrix. 
3. Initial Value Techniques for Correcting an Approximate Eigenvalue. The methods we consider in this section have as a common feature that they seek to correct $\lambda$ by using an approximate eigenvector $\mathbf{V}$ which is obtained as the solution to an initial value problem. To compute $\mathbf{V}$ we choose $V_{0}$ arbitrarily, then

and

$$
b_{0} V_{1}=-\left(h^{2} \lambda q_{0}+a_{0}\right) V_{0},
$$

$$
b_{i} V_{i+1}=-\left(h^{2} \lambda q_{i}+a_{i}\right) V_{i}-c_{i} V_{i-1}, \quad i=2,3, \cdots, n-2 .
$$

It is important to note that by suitable choice of $V_{0}$ we can arrange for any one of the components of $\mathrm{V}$ to be fixed independent of the value of $\lambda$.

From equation (3.1) we see that the components of $A \mathrm{~V}$ are

$$
\begin{gathered}
b_{i} V_{i+1}+a_{i} V_{i}+c_{i} V_{i-1}=-h^{2} \lambda q_{i} V_{i}, \quad i=0,1, \cdots, n-2, \\
c_{n-1} V_{n-2}+a_{n-1} V_{n-1}=-h^{2} \lambda q_{n-1} V_{n-1}+\left(h^{2} \lambda q_{n-1}+a_{n-1}\right) V_{n-1}+c_{n-1} V_{n-2},
\end{gathered}
$$

so that $\mathbf{V}$ also satisfies

$$
\left(A+h^{2} \lambda Q\right) \mathbf{V}=\beta \mathbf{e}_{n}
$$

where $\mathbf{e}_{n}$ is a vector with 1 in the $n$th place, and zero in all other positions. Also $\beta$ vanishes whenever $\lambda$ is an eigenvalue of (2.6) so that $\beta(\lambda)$ is proportional to the characteristic polynomial of (2.6).

One method of improving $\lambda$ is to calculate the Rayleigh quotient of equation (2.7) using the approximate eigenvector $\mathrm{V}$. In this case the Rayleigh quotient is

$$
\frac{\mathbf{V}^{\prime} D A \mathbf{V}}{\mathbf{V}^{\prime} D Q \mathbf{V}}=\frac{\mathbf{V}^{* \prime} A \mathbf{V}}{\mathbf{V}^{* \prime} Q \mathbf{V}} \quad \text { where } \quad \mathbf{V}^{*}=D \mathbf{V}
$$

Making use of equation (3.2) we have

$$
\frac{\mathrm{V}^{* \prime} A \mathrm{~V}}{\mathrm{~V}^{* \prime} Q \mathrm{~V}}=-h^{2} \lambda+\frac{\beta V_{n-1}^{*}}{\mathrm{~V}^{* \prime} Q \mathrm{~V}}
$$

It will be seen that (3.3) gives the correction to $\lambda$ in a very economical form. Another possibility is to apply Newton's method to find the zeros of $\beta(\lambda)$. This suggests that $\lambda-\beta / \frac{d \beta}{d \lambda}$ is a better approximation to the desired eigenvalue. We can calculate $\frac{d \beta}{d \lambda}$ by differentiating equation (3.2). This gives us

$$
\left(A+h^{2} \lambda Q\right) \frac{d \mathbf{V}}{d \lambda}+h^{2} Q \mathbf{V}=\frac{d \beta}{d \lambda} \mathbf{e}_{n}
$$

If we take the scalar product of equation (3.4) with $\mathbf{e}_{n}$ we have

$$
c_{n-1} \frac{d V_{n-2}}{d \lambda}+\left(a_{n-1}+h^{2} \lambda q_{n-1}\right) \frac{d V_{n-1}}{d \lambda}+h^{2} q_{n-1} V_{n-1}=\frac{d \beta}{d \lambda}
$$

This equation forms the basis of the procedure described by Fox (see [3], Chapter 8 , and references given there). He calculates $\frac{d \mathbf{V}}{d \lambda}$ by choosing the scale of $\mathbf{V}$ so that $V_{0}$ in equation (3.1) is independent of $\lambda$. This gives $\frac{d V_{0}}{d \lambda}=0$, and we have 


$$
\begin{gathered}
b_{0} \frac{d V_{1}}{d \lambda}=-\left(h^{2} \lambda q_{0}+a_{0}\right) \frac{d V_{0}}{d \lambda}-h^{2} q_{0} V_{0}=-h^{2} q_{0} V_{0}, \\
b_{i} \frac{d V_{i+1}}{d \lambda}=-\left(h^{2} \lambda q_{i}+a_{i}\right) \frac{d V_{i}}{d \lambda}-c_{i} \frac{d V_{i-1}}{d \lambda}-h^{2} q_{i} V_{i}, \\
i=1,2, \cdots, n-2 .
\end{gathered}
$$

However, if we take the scalar product of equation (3.4) with $\mathbf{V}^{*}$ we find, on noting that $\mathbf{V}^{*}$ satisfies

$$
\left(A^{\prime}+h^{2} \lambda Q\right) \mathbf{V}^{*}=\beta D \mathbf{e}_{n}=d_{n-1} \beta \mathbf{e}_{n},
$$

that

$$
d_{n-1} \beta \mathbf{e}_{n}{ }^{\prime} \frac{d \mathbf{V}}{d \lambda}+h^{2} \mathbf{V}^{* \prime} \mathbf{Q V}=V_{n-1}^{*} \frac{d \beta}{d \lambda}
$$

The correction formula given by Cooley [1] can be derived from (3.7) by choosing the scale of $\mathbf{V}$ so that $V_{n-1}$ is independent of $\lambda$. Then $\frac{d V_{n-1}}{d \lambda}=\mathbf{e}_{n}{ }^{\prime} \frac{d \mathbf{V}}{d \lambda}=0$, and $\frac{d \beta}{d \lambda}=h^{2} \frac{\mathbf{V}^{* \prime} Q \mathbf{V}}{\mathbf{V}_{n-1}^{*}}$. The correction to $\lambda$ is $-h^{-2} \frac{V_{n-1}^{*} \beta}{\mathbf{V}^{* \prime} Q \mathbf{V}}$. It is identical with that given by the Rayleigh quotient (3.3).

It does not follow from this result that Fox's procedure is equivalent to an application of the Rayleigh quotient because the value of $\beta$ is dependent on the way in which the scale is fixed in each method (although the correction to $\lambda$ in each case is independent of the choice of scale), and the scale factors are in general different functions of $\lambda$. We make this point clear by relating the principal minors of the determinant of (2.6) to the successive terms in the recurrence (3.1).

The principal minors of (2.6) satisfy the recurrence

$$
\begin{aligned}
& D_{0}=1 \\
& D_{1}=\left(a_{0}+h^{2} \lambda q_{0}\right) D_{0} \\
& D_{i+1}=\left(a_{i}+h^{2} \lambda q_{i}\right) D_{i}-c_{i} b_{i-1} D_{i-1} \\
& D \quad=\operatorname{det}\left(A+h^{2} \lambda Q\right)=\left(a_{n-1}+h^{2} \lambda q_{n-1}\right) D_{n-1}-c_{n-1} b_{n-2} D_{n-2} .
\end{aligned}
$$

If we put $V_{0}=s D_{0}$ where $s$ is the scale factor, then

and

$$
\begin{aligned}
b_{0} V_{1} & =-s D_{1}, \\
b_{1} V_{2} & =-\left(h^{2} \lambda q_{1}+a_{1}\right) V_{1}-c_{1} V_{0} \\
& =\left(h^{2} \lambda q_{1}+a_{1}\right) \frac{s D_{1}}{b_{0}}-c_{1} s D_{0}=\frac{s D_{2}}{b_{0}},
\end{aligned}
$$

so that $b_{0} b_{1} \dot{V}_{2}=s D_{2}$. This procedure can be continued, giving

$$
s D_{i}=(-1)^{i} b_{0} b_{1} \cdots b_{i-1} V_{i}
$$

and, in particular,

$$
D=\operatorname{det}\left(A+h^{2} \lambda Q\right)=(-1)^{n-1} \frac{b_{0} b_{1} \cdots b_{n-2}}{s} \beta .
$$


If $V_{0}$ is fixed independent of $\lambda$ then $s$ is a constant, and $\beta$ differs from the characteristic polynomial only by a constant multiplier. Therefore, Fox's method is equivalent to applying Newton's method to the characteristic polynomial. If $V_{n-1}$ is fixed independent of $\lambda$ then, by $(3.8), s$ is inversely proportional to $D_{n-1}$ so that $\beta$ differs from $D / D_{n-1}$ only by a constant multiplier. Now, by a well-known result for Sturm sequences, the zeros of $D_{n-1}$ interlace those of $D$ so that for the Rayleigh quotient to give a useful correction to $\lambda$ it is necessary that $\lambda$ lie inside the interval containing the desired eigenvalue bounded by the pair of adjacent zeros of $D_{n-1}$. No such restriction applies to Fox's iteration.

The results obtained in this section can readily be extended to the Numerov difference approximation (equation (2.10)). The initial value procedure gives a vector $\mathbf{V}$ satisfying

$$
\left(M+h^{2} \lambda N\right) \mathbf{V}=\beta \mathbf{e}_{n},
$$

and the only change is in the definition of $\mathrm{V}^{*}$ which we take as the solution of

$$
\left(M^{\prime}+h^{2} \lambda N^{\prime}\right) \mathbf{V}^{*}=\beta^{*} \mathbf{e}_{n} .
$$

We can calculate $\mathrm{V}^{*}$ by the initial value procedure. The value of $\beta^{*}$ then depends on the choice of scale. The Rayleigh quotient is given by

$$
\frac{\mathrm{V}^{* \prime} M \mathrm{~V}}{\mathrm{~V}^{* \prime} N \mathrm{~V}}=-h^{2} \lambda+\frac{\beta V_{n}^{*}}{\mathrm{~V}^{* \prime} N \mathrm{~V}}
$$

and this determines the correction to $\lambda$. The application of Newton's method proceeds exactly as before.

4. The Rayleigh Quotient Exemplified. In this section we exemplify the use of the Rayleigh quotient by applying it to calculate the fundamental eigenvalue of the standard finite difference approximation (equation (2.3)) to the differential equation

$$
\frac{d^{2} y}{d x^{2}}+\frac{1}{x} \frac{d y}{d x}+\lambda y=0
$$

with the boundary conditions $\frac{d y(0)}{d x}=0, y(1)=0$. As $\frac{d y}{d x}=0$ when $x=0$ we see that the required solution to (4.1) satisfies

$$
2 \frac{d^{2} y}{d x^{2}}+\lambda y=0
$$

at this point. Also it is an even function about $x=0$ so that the finite difference approximation (equation (2.5)) to the boundary condition $\frac{d y(0)}{d x}=0$ is exact.

In this case $Q$ is the unit matrix. Taking $n=4, h=\frac{1}{4}$ we find that $A$ is the matrix

$$
\left[\begin{array}{cccc}
-4 & 4 & & \\
\frac{1}{2} & -2 & \frac{3}{2} & \\
& \frac{3}{4} & -2 & \frac{5}{4} \\
& & \frac{5}{6} & -2
\end{array}\right]
$$


It is readily verified that a suitable diagonal matrix such that $D A$ is symmetric is

$$
D=\left[\begin{array}{llll}
\frac{1}{2} & & & \\
& 4 & & \\
& & 8 & \\
& & & 12
\end{array}\right]
$$

In the table that follows we give the result of the computation. We use equation (3.1) to run forward the approximate eigenvectors (rounding to six decimals). The corrections to $\lambda$ are shown in the final column.

\begin{tabular}{ccccccr}
$h^{2} \lambda$ & $y(0)$ & $y\left(\frac{1}{4}\right)$ & $y\left(\frac{1}{2}\right)$ & $y\left(\frac{3}{4}\right)$ & $\beta$ & \multicolumn{1}{c}{$\frac{\beta V_{n}{ }^{*}}{\mathrm{~V}^{* \prime} \mathbf{V}}$} \\
& & & & & & -.040742 \\
.312500 & 1.000000 & .921875 & .703776 & .396973 & -.083412 & -.004058 \\
.353242 & 1.000000 & .911689 & .667555 & .332428 & .008867 & .004005 \\
.349184 & 1.000000 & .912704 & .671138 & .338718 & .000120 & .000055 \\
.349129 & 1.090000 & .912718 & .671187 & .338804 & .000001 & -
\end{tabular}

The quadratic nature of the convergence is clearly indicated.

For this example the Rayleigh quotient requires about half as much arithmetic as Fox's method. It has another advantage for the vector $V^{*}$ is readily to hand if it is required to compute an estimate of the deviation of the computed $\lambda$ from the exact eigenvalue of the differential equation by using the difference correction (see reference [3]). Returning to our example we set

$$
\mathcal{C} y_{i}=\frac{1}{12}\left\{\frac{2}{i} \mu \delta^{3}+\delta^{4}\right\} y_{i}
$$

except when $i=0$ when

$$
\mathfrak{C} y_{0}=\frac{1}{6} \delta^{4} y_{0}
$$

As the finite difference boundary conditions are exact in this case an estimate of the correction to $\lambda$ is given by

\begin{tabular}{|c|c|c|c|c|c|c|}
\hline$i$ & $y_{i}$ & $\delta$ & $\delta^{2}$ & $\delta^{3}$ & $\mu \delta^{3}$ & $\delta^{4}$ \\
\hline-1 & .912718 & & & & & \\
\hline 0 & 1.000000 & - 087282 & -.174564 & 020315 & 0 & \\
\hline 1 & .912718 & .001202 & -.154249 & & .041856 & .043082 \\
\hline 2 & .671187 & $\begin{array}{l}-.241531 \\
-\quad 332383\end{array}$ & -.090852 & 084431 & .073914 & .021034 \\
\hline 3 & .338804 & -.338804 & -.006421 & & .083071 & -.002720 \\
\hline 4 & -.263514 & & .075290 & & & \\
\hline
\end{tabular}

$$
h^{2} \Delta \lambda=\frac{\mathbf{V}^{* \prime} \mathbf{C y}}{\mathrm{V}^{* \prime} \mathbf{y}}
$$

The difference table is

Equation (4.3) gives us $h^{2} \Delta \lambda=.01184$. The basic difference equation gives $\lambda=$ 5.59. Taking into account the difference correction gives $\lambda=5.776$ which compares much more favourably with the exact value $(\lambda=5.781 \cdots)$. 
5. The Problem of Stability. The crucial factor to be considered in suggesting the Rayleigh quotient procedure as a general routine is the stability of the calculation of the approximate eigenvector; it is well known that we cannot use the procedure summarized in equation (3.1) in general as the resulting vector may well differ substantially from the exact eigenvector. There is, however, numerical evidence that Fox's iteration works (and gives quadratic convergence) for the eigenvalue at least in some cases of instability in the calculation of the vector (see [4]). It is doubtful if the Rayleigh quotient iteration can be successful in these circumstances, and it is interesting that the numerical evidence would seem to indicate that there is a root of $D_{n-1}$ very close to the desired root of $D$ in the direction of increasing $\lambda$. In many cases this evidence can be supported by heuristic argument.

In solving problems associated with second-order differential equations one often knows beforehand that instability is likely for part of the range for which the solution is defined. For example, the desired solution may have the character of an exponential decay in some region where the second solution to the differential equation is changing rapidly, and we may anticipate that the solutions of our second-order difference equation mirror this behavior. However, if instability is likely when running forward due to a rapidly increasing spurious solution then we may expect stability if we reverse the direction of the computation. This device works well within the limits of its applicability.

With little loss of convenience we may march the approximate eigenvector back from the last row of the matrix as well as forward from the first row. If we match the computations by making their $r$ th components agree then we have solved

$$
\left(A+h^{2} \lambda Q\right) \mathbf{V}=\beta \mathbf{e}_{r}
$$

where $1 \leqq r \leqq n$. The analysis of Section 3 can be applied to (5.1). We find that Newton's method applied to $\beta(\lambda)$ with $v_{0}$ fixed independent of $\lambda$ (Fox's correction) is equivalent to applying Newton's method to $D / E_{n-r+1}$, while the Rayleigh quotient is equivalent to Newton's method applied to $D / D_{r-1} E_{n-r+1}$. Here $E_{n-r+1}$ is the principal minor with diagonal elements $\left(a_{r}+h^{2} \lambda q_{r}\right), \cdots,\left(a_{n-1}+h^{2} \lambda q_{n-1}\right)$.

If it is necessary to do more than march from both ends to achieve stability then the initial value techniques lose much of their simplicity. In this case we are forced to solve an equation of the form

$$
\left(A+h^{2} \lambda Q\right) \mathbf{V}=\mathbf{k}
$$

where we have considerable freedom in the choice of $\mathbf{k}$. The calculation of an eigenvector of $A+h^{2} \lambda Q$ from (5.2) has been discussed in detail by Wilkinson in reference [5]. In particular, he suggests that $\mathbf{k}$ at any stage should be the approximate eigenvector calculated in the previous iteration.

In considering finite difference approximations to eigenvalues of higher-order differential equations we find that $A$ is no longer tridiagonal, and that the Rayleigh quotient procedure cannot be readily combined with an initial value procedure for the desired eigenvector. Fox's procedure can be modified to apply in this case [3] and [4]. The cost is the addition of further variational equations similar to (3.6). However, the Rayleigh quotient is defined whenever the approximate vectors 
$\mathbf{V}$ and $\mathbf{V}^{*}$ are available, and Wilkinson's experience in [5] suggests the iteration

$$
\begin{aligned}
\left(A+h^{2} \lambda_{s-1} Q\right) \mathbf{V}_{s} & =\mathbf{V}_{s-1}, \\
\left(A^{\prime}+h^{2} \lambda_{s-1} Q\right) \mathbf{V}_{s}^{*} & =\mathbf{V}_{s-1}^{*}, \\
h^{2} \lambda_{s} & =h^{2} \lambda_{s-1}-\frac{\mathbf{V}_{s}^{* \prime} \mathbf{V}_{s-1}}{\mathbf{V}_{s}^{* \prime} Q \mathbf{V}_{s}} .
\end{aligned}
$$

Setting $A+h^{2} \lambda Q=L U$, then

$$
\mathbf{V}_{s}=U^{-1} L^{-1} \mathbf{V}_{s-1}
$$

and

$$
\mathbf{V}_{s}^{*}=\left(L^{-1}\right)^{\prime}\left(U^{-1}\right)^{\prime} \mathbf{V}_{s-1}^{*}
$$

so that each iteration (5.3) requires only one matrix factorization. For numerical stability it would be necessary to use the method of triangular factorization with interchanges given by Wilkinson in $[6, \mathrm{p} .18-19]$.

6. A Computational Procedure. This paper is based on experience gained over several years in solving differential equation eigenvalue problems which arise in the theory of the propagation of sound in the ocean treated as a layered fluid medium (the theoretical background to this work has been described in [7]). Here one boundary is taken at an infinite depth (which is treated as very great in the numerical work); the differential equations have, in general, mixed spectra. Our concern has been to calculate the eigenvalues and eigenfunctions in the discrete spectrum.

The differential equation has the form

$$
\frac{d^{2} y}{d x^{2}}+(\lambda+q(x)) y=0
$$

so that the Numerov difference approximation (equation (2.10)) is applicable, and we solve this in the following stages.

(I) We begin by using the standard finite difference approximation. We use the Sturm count procedure to roughly isolate an eigenvalue; and we then use the Rayleigh quotient formula to obtain a quadratically convergent iteration to it. The use of the standard equation instead of the Numerov equation at this stage approximately halves our arithmetic.

(II) We calculate an $\mathrm{O}\left(h^{2}\right)$ improvement to this value of $\lambda$ using the difference correction technique exactly as described in Section 4 . This gives an eigenvalue having the same kind of order of magnitude deviation from the exact value as does the corresponding eigenvalue of (2.10).

(III) We use the Rayleigh quotient iteration (3.12) to calculate the accurate eigenvalue of equation (2.10) from the value of $\lambda$ obtained in stage (II). We also obtain a useful improvement in the accuracy of the eigenvector. If further refinement is required we can calculate an improvement to $\lambda$ (and, if desired, to y) by a further stage of difference correction.

Using values of $n$ up to 100 we have been able to obtain stable computations by marching forward the calculation of the eigenvector from each boundary point, and matching at a suitably chosen interior point. There is physical evidence to 
support the use of these tactics because regions of low acoustic pressure can occur near the surface of the ocean as well as at great depth. In no case have more than two iterations been required in stage (III) of the computation. Comparison of the values of $\lambda$ obtained at the various stages of the computation gives us useful information about the accuracy of our approximations.

University of Reading

England

1. J. W. Cooley, "Improved formula for solving central fields," Math. Comp., v. 15, 1961, p. $363-374$.

2. A. S. Housenolder, Principles of Numerical Analysis, McGraw-Hill, 1953.

3. L. Fox, The Numerical Solution of Two-point Boundary Value Problems, Oxford, 1957.

4. L. Fox, "Some numerical experiments with eigenvalue problems in ordinary differential equations," included in Boundary Problems in Differential Equations (Editor R. E. Langer), University of Wisconsin Press, 1960.

5. J. H. WILKINSON, "Calculation of eigenvectors of codiagonal matrices," Comput. J., v. 1 , 1958 , p. $90-96$.

6. National Physical Laboratory, Modern Computing Methods, Notes on Applied Science No. 16, Her Majesty's Stationery Office, London, 1961.

7. M. R. OsBorse, "The propagation of sound in a layered fluid medium," Quart. $J$. Mech. Appl. Math., v. 13, n. 4, 1960, p. 472-86. 